\title{
Sınır Koşulunda Öz Parametre Bulunduran Bir Sturm-Liouville Operatörü İçin Ters Nodal Problem
}

\author{
Inverse Nodal Problem for A Sturm-Liouville Operator with Eigenparameter in the \\ Boundary Condition
}

\section{Sertaç GÖKTAŞ*}

Mersin Üniversitesi, Fen-Edebiyat Fakültesi, Matematik Bölümü, 33343, Mersin

$\ddot{O} \mathbf{z}$

$\mathrm{Bu}$ çalışmada, sınır koşulunda öz parametre ve ikinci derece diferensiyel denkleminde birden fazla potansiyel bulunduran bir Sturm- Liouville problemi ele alınmıştır. Prüfer dönüşümü yardımıyla bu problemin özdeğerlerinin ve nodal parametrelerinin asimptotik formülleri bulunmuştur. Ayrıca, potansiyel fonksiyonlar için bir yapılandırma formülü elde edilmiștir.

Anahtar kelimeler: Nodal Noktalar, Nodal Uzunluklar, Potansiyel Fonksiyon, Prüfer Dönüşümü

\begin{abstract}
In this study, Sturm-Liouville problem with eigenparameter in the boundary condition and more than one potential in the second order differential equation is considered. Asymptotic formulas of the eigenvalues and nodal parameters of this problem are found by Prüfer substitution. In addition, a reconstruction formula is obtained for potential functions.
\end{abstract}

Keywords: Nodal Points, Nodal Lengths, Potential Function, Prüfer Substitution 


\section{Giriş}

Lineer diferansiyel operatörlerin spektral teorisinde, düz problemin ve ters problemin çeşitli özellikleri birçok yazar tarafindan araştırılmıştır. Burada, düz problem: diferansiyel operatörlerin öz değerlerinin ve bu öz değerlere karşılık gelen öz fonksiyonların belirlenmesi problemi; ters problem ise özdeğer, özfonksiyon, saçılma verileri, normlaştırıcı sabitler, özdeğerlerin sıfırları gibi spektral karakteristikler kullanılarak denklemin katsayı fonksiyonlarının bulunması yani kısaca lineer operatörün biçiminin bulunması problemi olarak tanımlanır. $\mathrm{Bu}$ iki problemden ikincisi yani spektral analizin ters problemleri, matematik, fizik, mekanik, elektronik, jeofizik, meteoroloji, sismoloji, tıp ve bu gibi başka doğa bilimlerinde ortaya çıkan önemli problemlerin çözülmesinde önemli role sahiptir (Çakır, 2007).

Sturm-Liouville operatörü için ters problemlerin gelişmesinde önemli katkı Ambartsumyan (1929) tarafindan formulüze edilen ve incelenen çalıșma ile olmuştur. Fakat, bu çalışmanın aksine özdeğerlerin bir kümesinin operatörün biçimini belirlemesinde yeterli olmadığı anlaşıldı. Örneğin; Ambarsumyan'ın özdeğerlerin tek bir kümesinin bilinmesi" koşuluna ek olarak, Borg (1946) özdeğerlerin ikinci bir kümesinin bilinmesi veya $q(x)=q(1-x) \quad$ koşulunun sağlanmasının operatörün biçimini belirlemesinde yeterli olduğunu; Gel'fand ve Levitan (1951) normlaştırıcı sabitler kümesi kullanarak potansiyel fonksiyonun tek olarak belirlenebileceğini; Hoschtadt (1973) öz fonksiyonların indis kümesi üzerinde bir mutlak toplam olarak potansiyel fonksiyonun elde edilebileceğini gösterdiler.

Son y1llarda, ters problemlerin yeni bir sinifi olan ters nodal problemler teorisi yazarların dikkatini oldukça çekmiştir. Ters nodal problem ilk olarak McLaughlin (1988) tarafindan ele alındı. SturmLiouville probleminde potansiyel fonksiyonu tek olarak belirlemek için sadece nodal noktaların (öz fonksiyonların sifirlarının) bilgisinin yeterli olduğu ispatlandı (McLaughlin, 1988; Hald ve McLaughlin, 1989). Daha sonra, bazı yazarlar tarafından dikkate değer bazı sonuçlar elde edilmiştir. Örneğin, bazı yazarlar çalışmalarında nodal noktalar yardımiyla potansiyel fonksiyonu ve türevlerini yeniden yapılandırmıştır (Chen vd., 2002; Koyunbakan ve Panakhov, 2007; Koyunbakan ve Y1lmaz, 2008; Koyunbakan, 2009, 2011; Yang, 2014; Pinasco ve Scarola, 2015). Bu çalışmalara ek olarak bazı yazarlar sınır koşullarının öz parametreye bağlı olması durumunda ters nodal problemi ele aldilar (Browne ve Sleeman, 1996; Y1lmaz ve Koyunbakan, 2010; Panakhov vd., 2010; Keskin ve Özkan, 2017; Şen, 2017, 2018).

Sturm-Liouville operatörünün birden fazla potansiyel fonksiyonu kapsaması bakımından klasik Sturm-Liouville operatöründen farkl1 ters nodal problemler bazı yazarlar tarafindan çalışılmıştır (Guseinov ve Nabiev, 2000; Guseinov v.d. 2000; Nabiev, 2010; Goktas vd., 2018). Ayrıca, Kaplan (2019) bu tip bir operatörü ayrılabilir sınır şartlarıyla birlikte göz önüne alarak nodal noktalar yardımıyla ters problemin çözümüne ulaşmıştır.

Bu çalışmada, $0 \leq x \leq \pi$ aralığında aşağıdaki problemi ele alacağız:

$-y^{\prime \prime}+\left\{q_{0}(x)+\lambda q_{1}(x)+\lambda^{2} q_{2}(x)\right\} y=\lambda^{6} y$,

$y(0)=0$,

$y^{\prime}(\pi)+\lambda^{2} y(\pi)=0$.

Burada, $\lambda$ bir öz parametre ve $q_{k-1}(x), k=\overline{1,3}$ fonksiyonları $[0, \pi]$ aralı̆̆ında reel değerli sürekli fonksiyonlardır. Bu çalışmadaki amaç, Prüfer dönüşümü yardımıyla sınır koşullarının birinde öz parametre bulunduran (1)-(3) probleminin potansiyel fonksiyonları için bir yapılandırma formülü elde etmektir.

(1)-(3) probleminin $\lambda_{n}$ öz değerlerine karşılık gelen $y_{n}(x)=y\left(x, \lambda_{n}\right)$ öz fonksiyonlarının sıfırlarının kümesi olan $\left\{x_{j}^{n}\right\}_{j=1}^{n-1}, n \geq 2$ kümesi nodal nokta kümesi diye adlandırılır. $l_{j}^{n}=x_{j+1}^{n}-x_{j}^{n}, j=\overline{1, n-1}, n \geq 2$ değerine ise nodal uzunluk adı verilir.

\section{2. Özdeğer ve Nodal Parametreler için Asimptotik Formüller}

Bu bölümde, (1)-(3) probleminin öz değerleri ve nodal parametreleri (nodal noktalar ve nodal uzunluklar) için asimptotik formüller verilmiştir. Bu formüllerin hesaplanmasında Prüfer dönüşümü kullanıldı. (1) 
denkleminin aşikar olmayan $y(x)$ çözümü için bu dönüşüm aşağıdaki formda yazılır:

$$
\begin{aligned}
& y(x)=s(x) \sin \left(\lambda^{3} \theta(x)\right), \\
& y^{\prime}(x)=\lambda^{3} s(x) \cos \left(\lambda^{3} \theta(x)\right) \\
& \text { veya } \\
& \frac{y^{\prime}(x)}{y(x)}=\lambda^{3} \cot \left(\lambda^{3} \theta(x)\right) .
\end{aligned}
$$

Burada, $s(x)$ fonksiyonu genlik(amplitude) ve $\theta(x)$ fonksiyonu ise faz(phase) olarak adlandir1lır

(Birkhoff ve Rota, 1982).

Diğer taraftan,

$$
\frac{y^{\prime \prime}(x)}{y(x)}=\left(\frac{y^{\prime}(x)}{y(x)}\right)^{\prime}+\left(\frac{y^{\prime}(x)}{y(x)}\right)^{2}
$$

eşitliği yazılabilir. Son eşitlik ile birlikte (6) eşitliği (1) denkleminde göz önüne alınırsa

$$
\theta^{\prime}(x)=1-\frac{1}{\lambda^{6}}\left(q_{0}(x)+\lambda q_{1}(x)+\lambda^{2} q_{2}(x)\right) \sin ^{2}\left(\lambda^{3} \theta(x)\right)
$$

formülü elde edilir.

$\mathrm{Bu}$ çalışma boyunca, (1)-(3) problemi için kurulan teoremlerin ispatında $\lambda=\lambda_{n}, \quad n \in \mathbb{N}$ olduğu varsay1lacaktır (Shukurov, 2009).

Teorem 2.1. $n \rightarrow \infty$ iken (1)-(3) probleminin öz değerlerinin asimptotik formülü aşağıdaki biçimdedir:

$$
\lambda_{n}^{3}=n+\frac{1}{2}+O\left(\frac{1}{\sqrt[3]{n}}\right)
$$

İspat. (2) sınır koşulu ve (4) ten $\theta(0)=0$ elde edilir. Buna ek olarak, (3) sınır koşulundan $\frac{y^{\prime}(\pi)}{y(\pi)}=-\lambda_{n}^{2}$ ve $x=\pi$ için (4), (5) eşitliklerinden $\frac{y^{\prime}(\pi)}{y(\pi)}=\lambda_{n}^{3} \cot \left(\lambda_{n}^{3} \theta(\pi)\right)$ elde edilir. Bu son iki eşitlik birlikte dikkate alınırsa

$$
\theta(\pi)=\frac{1}{\lambda_{n}^{3}} \operatorname{arccot}\left(-\frac{1}{\lambda_{n}}\right)=\frac{1}{\lambda_{n}^{3}}\left(\left(n+\frac{1}{2}\right) \pi+O\left(\frac{1}{\lambda_{n}}\right)\right)=\frac{\left(n+\frac{1}{2}\right) \pi}{\lambda_{n}^{3}}+O\left(\frac{1}{\lambda_{n}^{4}}\right)
$$

bulunur.

$[0, \pi]$ aralığında (7) eşitliğinin her iki tarafının $x$ e göre integrali alınırsa

$$
\begin{aligned}
\frac{\left(n+\frac{1}{2}\right) \pi}{\lambda_{n}^{3}}+O\left(\frac{1}{\lambda_{n}^{4}}\right) & =\pi-\frac{1}{\lambda_{n}^{6}} \int_{0}^{\pi} q_{0}(x) \sin ^{2}\left(\lambda_{n}^{3} \theta(x)\right) d x-\frac{1}{\lambda_{n}^{5}} \int_{0}^{\pi} q_{1}(x) \sin ^{2}\left(\lambda_{n}^{3} \theta(x)\right) d x \\
& -\frac{1}{\lambda_{n}^{4}} \int_{0}^{\pi} q_{2}(x) \sin ^{2}\left(\lambda_{n}^{3} \theta(x)\right) d x \\
& =\pi-\frac{1}{2 \lambda_{n}^{6}} \int_{0}^{\pi} q_{0}(x) d x-\frac{1}{2 \lambda_{n}^{5}} \int_{0}^{\pi} q_{1}(x) d x-\frac{1}{2 \lambda_{n}^{4}} \int_{0}^{\pi} q_{2}(x) d x \\
& +\frac{1}{2 \lambda_{n}^{6}} \int_{0}^{\pi} q_{0}(x) \cos \left(2 \lambda_{n}^{3} \theta(x)\right) d x+\frac{1}{2 \lambda_{n}^{5}} \int_{0}^{\pi} q_{1}(x) \cos \left(2 \lambda_{n}^{3} \theta(x)\right) d x \\
& +\frac{1}{2 \lambda_{n}^{4}} \int_{0}^{\pi} q_{2}(x) \cos \left(2 \lambda_{n}^{3} \theta(x)\right) d x .
\end{aligned}
$$


bulunur. $\mathrm{Bu}$ son ifadede,

$\cos \left(2 \lambda_{n}^{3} \theta(x)\right)=\frac{1}{2 \lambda_{n}^{3} \theta^{\prime}(x)} \frac{d}{d x}\left[\sin \left(2 \lambda_{n}^{3} \theta(x)\right)\right]$

formülü dikkate alınırsa

$$
\begin{aligned}
\frac{\left(n+\frac{1}{2}\right) \pi}{\lambda_{n}^{3}}+O\left(\frac{1}{\lambda_{n}^{4}}\right) & =\pi-\sum_{m=0}^{2} \frac{1}{2 \lambda_{n}^{6-m}} \int_{0}^{\pi} q_{m}(x) d x \\
& +\sum_{m=0}^{2} \frac{1}{2 \lambda_{n}^{6-m}} \int_{0}^{\pi} \frac{q_{m}(x)}{2 \lambda_{n}^{3} \theta^{\prime}(x)} \frac{d}{d x}\left[\sin \left(2 \lambda_{n}^{3} \theta(x)\right)\right] d x .
\end{aligned}
$$

eşitliği elde edilir. (9) eşitliğinin sağındaki son üç integrale kısmi integrasyon metodu uygulansın:

$$
\begin{aligned}
\int_{0}^{\pi} \frac{q_{0}(x)}{2 \lambda_{n}^{3} \theta^{\prime}(x)} \frac{d}{d x}\left[\sin \left(2 \lambda_{n}^{3} \theta(x)\right)\right] d x & =\left.\frac{q_{0}(x)}{2 \lambda_{n}^{3} \theta^{\prime}(x)} \sin \left(2 \lambda_{n}^{3} \theta(x)\right)\right|_{x=0} ^{x=\pi}-\frac{1}{2 \lambda_{n}^{3}} \int_{0}^{\pi} \sin \left(2 \lambda_{n}^{3} \theta(x)\right) d\left(\frac{q_{0}(x)}{\theta^{\prime}(x)}\right) \\
& =O\left(\frac{1}{\lambda_{n}^{3}}\right) .
\end{aligned}
$$

Benzer şekilde,

$\int_{0}^{\pi} \frac{q_{1}(x)}{2 \lambda_{n}^{3} \theta^{\prime}(x)} \frac{d}{d x}\left[\sin \left(2 \lambda_{n}^{3} \theta(x)\right)\right] d x=O\left(\frac{1}{\lambda_{n}^{3}}\right)$ ve $\int_{0}^{\pi} \frac{q_{2}(x)}{2 \lambda_{n}^{3} \theta^{\prime}(x)} \frac{d}{d x}\left[\sin \left(2 \lambda_{n}^{3} \theta(x)\right)\right] d x=O\left(\frac{1}{\lambda_{n}^{3}}\right)$.

Hesaplanan bu son üç değer (9) eşitliğinde göz önüne alınırsa

$\frac{\left(n+\frac{1}{2}\right) \pi}{\lambda_{n}^{3}}+O\left(\frac{1}{\lambda_{n}^{4}}\right)=\pi-\sum_{m=0}^{2} \frac{1}{2 \lambda_{n}^{6-m}} \int_{0}^{\pi} q_{m}(x) d x+O\left(\frac{1}{\lambda_{n}^{7}}\right)$

veya

$\frac{\left(n+\frac{1}{2}\right) \pi}{\lambda_{n}^{3}}=\pi+O\left(\frac{1}{\lambda_{n}^{4}}\right)$

elde edilir. Buradan, $\frac{1}{1 \mp O(h(n))}=1+O(h(n))$ özelliği yardımıyla

$\lambda_{n}^{3}=\left(n+\frac{1}{2}\right)\left(1+O\left(\frac{1}{\lambda_{n}^{4}}\right)\right)$

formülü elde edilir. Diğer taraftan, bu son eşitlikte $n \rightarrow \infty$ için limit alınrsa $\lambda_{n} \approx\left(n+\frac{1}{2}\right)^{\frac{1}{3}}$ yaklaşık değeri bulunur. $\lambda_{n}$ nın bu yaklaşık değeriyle birlikte, teorem 2.1 ispatlanmış olur.

Teorem 2.2. $n \rightarrow \infty$ iken (1)-(3) probleminin nodal noktalart için asimptotik formül aşağıdaki biçimdedir:

$x_{j}^{n}=\frac{j \pi}{\lambda_{n}^{3}}+\sum_{m=0}^{2} \frac{1}{2 \lambda_{n}^{6-m}} \int_{0}^{x_{j}^{n}} q_{m}(t) d t+O\left(\frac{1}{\lambda_{n}^{7}}\right)$.

İspat. $x=x_{j}^{n}$ olduğunu varsayalım. Bu durumda, $x=x_{j}^{n}$ lar nodal noktalar (öz fonksiyonların sıfırları) olduğundan $y\left(x_{j}^{n}\right)=0$ dır. Bu durum, (4) eşitliğinde dikkate alınırsa $\theta\left(x_{j}^{n}\right)=\frac{j \pi}{\lambda_{n}^{3}}$ değeri elde edilir.

$\left[0, x_{j}^{n}\right]$ aralığında (7) eşitliğinin her iki tarafının $x$ e göre integrali alınırsa

$\frac{j \pi}{\lambda_{n}^{3}}=x_{j}^{n}-\sum_{m=0}^{2} \frac{1}{\lambda_{n}^{6-m}} \int_{0}^{x_{j}^{n}} q_{m}(t) \sin ^{2}\left(\lambda_{n}^{3} \theta(t)\right) d t$ 
veya

$$
\begin{aligned}
x_{j}^{n} & =\frac{j \pi}{\lambda_{n}^{3}}+\sum_{m=0}^{2} \frac{1}{2 \lambda_{n}^{6-m}} \int_{0}^{x_{j}^{n}} q_{m}(t) d t-\sum_{m=0}^{2} \frac{1}{2 \lambda_{n}^{6-m}} \int_{0}^{x_{j}^{n}} q_{m}(t) \cos \left(2 \lambda_{n}^{3} \theta(t)\right) d t \\
& =\frac{j \pi}{\lambda_{n}^{3}}+\sum_{m=0}^{2} \frac{1}{2 \lambda_{n}^{6-m}} \int_{0}^{x_{j}^{n}} q_{m}(t) d t-\sum_{m=0}^{2} \frac{1}{2 \lambda_{n}^{6-m}} \int_{0}^{x_{j}^{k}} \frac{q_{m}(t)}{2 \lambda_{n}^{3} \theta^{\prime}(t)} d\left[\sin \left(2 \lambda_{n}^{3} \theta(t)\right)\right]
\end{aligned}
$$

elde edilir.

(10) eşitliğinin sağındaki son üç integrale kısmi integrasyon metodu uygulanırsa, bu durumda $m=\overline{0,2}$ için

$$
\int_{0}^{x_{j}^{n}} \frac{q_{m}(t)}{2 \lambda_{n}^{3} \theta^{\prime}(t)} d\left[\sin \left(2 \lambda_{n}^{3} \theta(t)\right)\right]=-\frac{1}{2 \lambda_{n}^{3}} \int_{0}^{x_{j}^{n}} \sin \left(2 \lambda_{n}^{3} \theta(t)\right) d\left(\frac{q_{m}(t)}{\theta^{\prime}(t)}\right)=O\left(\frac{1}{\lambda_{n}^{3}}\right)
$$

bulunur. Bu nedenle

$$
\sum_{m=0}^{2} \frac{1}{2 \lambda_{n}^{6-m}} \int_{0}^{x_{j}^{n}} \frac{q_{m}(t)}{2 \lambda_{n}^{3} \theta^{\prime}(t)} d\left[\sin \left(2 \lambda_{n}^{3} \theta(t)\right)\right]=\frac{1}{\lambda_{n}^{6}} O\left(\frac{1}{\lambda_{n}^{3}}\right)+\frac{1}{\lambda_{n}^{5}} O\left(\frac{1}{\lambda_{n}^{3}}\right)+\frac{1}{\lambda_{n}^{4}} O\left(\frac{1}{\lambda_{n}^{3}}\right)=O\left(\frac{1}{\lambda_{n}^{7}}\right)
$$

dir. Bu son ifade (10) eşitliğinde dikkate alınırsa, aşağıdaki formül elde edilir:

$$
x_{j}^{n}=\frac{j \pi}{\lambda_{n}^{3}}+\sum_{m=0}^{2} \frac{1}{2 \lambda_{n}^{6-m}} \int_{0}^{x_{j}^{n}} q_{m}(t) d t+O\left(\frac{1}{\lambda_{n}^{7}}\right)
$$

Dolayısıyla, teorem 2.2 nin ispatı tamamlanır.

Teorem 2.3. $n \rightarrow \infty$ iken (1)-(3) probleminin nodal uzunlukları için asimptotik formül aşağıdaki biçimdedir:

$$
l_{j}^{n}=\frac{\pi}{\lambda_{n}^{3}}+\sum_{m=0}^{2} \frac{1}{2 \lambda_{n}^{6-m}} \int_{x_{j}^{n}}^{x_{j+1}^{n}} q_{m}(t) d t+O\left(\frac{1}{\lambda_{n}^{10}}\right)
$$

İspat. Yeterince büyük $n \in \mathbb{N}$ için, $\left[x_{j}^{n}, x_{j+1}^{n}\right]$ aralığında (7) eşitliğinin her iki tarafının $x=t$ e göre integrali alınırsa

$$
\begin{aligned}
\int_{x_{j}^{n}}^{x_{j+1}^{n}} \theta^{\prime}(t) d t & =\int_{x_{j}^{n}}^{x_{j+1}^{n}}\left(1-\sum_{m=0}^{2} \frac{1}{\lambda_{n}^{6-m}} q_{m}(t) \sin ^{2}\left(\lambda_{n}^{3} \theta(t)\right)\right) d t \\
\theta\left(x_{j+1}^{n}\right)-\theta\left(x_{j}^{n}\right) & =l_{j}^{n}-\sum_{m=0}^{2} \frac{1}{\lambda_{n}^{6-m}} \int_{x_{j}^{n}}^{x_{j+1}^{n}} q_{m}(t) \sin ^{2}\left(\lambda_{n}^{3} \theta(t)\right) d t \\
\frac{\pi}{\lambda_{n}^{3}} & =l_{j}^{n}-\sum_{m=0}^{2} \frac{1}{2 \lambda_{n}^{6-m}} \int_{x_{j}^{n}}^{x_{j+1}^{n}} q_{m}(t) d t+\sum_{m=0}^{2} \frac{1}{2 \lambda_{n}^{6-m}} \int_{x_{j}^{n}}^{x_{j+1}^{n}} q_{m}(t) \cos \left(2 \lambda_{n}^{3} \theta(t)\right) d t
\end{aligned}
$$

veya

$$
l_{j}^{n}=\frac{\pi}{\lambda_{n}^{3}}+\sum_{m=0}^{2} \frac{1}{2 \lambda_{n}^{6-m}} \int_{x_{j}^{n}}^{x_{j+1}^{n}} q_{m}(t) d t-\sum_{m=0}^{2} \frac{1}{2 \lambda_{n}^{6-m}} \int_{x_{j}^{n}}^{x_{j+1}^{n}} q_{m}(t) \cos \left(2 \lambda_{n}^{3} \theta(t)\right) d t
$$

elde edilir. 
$\mathrm{Bu}$ son eşitliğin sağ tarafindaki son eşitlikte son üç integral için yukarıdaki teoremlerde kullanılan benzer işlemler uygulanırsa, $m=\overline{0,2}$ için

$$
\begin{aligned}
\int_{x_{j}^{n}}^{x_{j+1}^{n}} q_{m}(t) \cos \left(2 \lambda_{n}^{3} \theta(t)\right) d & =\int_{x_{j}^{n}}^{x_{j+1}^{n}} \frac{q_{m}(t)}{2 \lambda_{n}^{3} \theta^{\prime}(t)} d\left[\sin \left(2 \lambda_{n}^{3} \theta(t)\right)\right] \\
& =-\frac{1}{2 \lambda_{n}^{6}} \int_{j \pi}^{(j+1) \pi} \sin (2 \xi) \frac{d}{d t}\left(\frac{q_{m}(t)}{\theta^{\prime}}\right) \frac{d \xi}{\theta^{\prime}} \\
& =O\left(\frac{1}{\lambda_{n}^{6}}\right)
\end{aligned}
$$

bulunur. Burada, $\xi=\lambda_{k}^{3} \theta(t)$ için $d t=\frac{d \xi}{\lambda_{n}^{3} \theta^{\prime}(t)}$ dir. Bu nedenle,

$$
\sum_{m=0}^{2} \frac{1}{2 \lambda_{n}^{6-m}} \int_{x_{j}^{n}}^{x_{j+1}^{n}} q_{m}(t) \cos \left(2 \lambda_{n}^{3} \theta(t)\right) d t=O\left(\frac{1}{\lambda_{n}^{10}}\right)
$$

dir.

Bu son eşitlik (12) eşitliğinde dikkate alınırsa, aşağıdaki formül elde edilir:

$$
l_{j}^{n}=\frac{\pi}{\lambda_{n}^{3}}+\sum_{m=0}^{2} \frac{1}{2 \lambda_{n}^{6-m}} \int_{x_{j}^{n}}^{x_{j+1}^{n}} q_{m}(t) d t+O\left(\frac{1}{\lambda_{n}^{10}}\right) .
$$

Dolayısıyla, teorem 2.3 ispatlanır.

Goktas vd., (2018) yapmış oldukları çalışmada kullanılan yönteme benzer olarak, $k=3$ için (11) eşitliği

$$
l_{j}^{n}=\frac{\pi}{\lambda_{n}^{k}}+\sum_{m=0}^{k-1} \frac{1}{2 \lambda_{n}^{2 k-m}} \int_{x_{j}^{n}}^{x_{j+1}^{n}} q_{m}(t) d t+O\left(\frac{1}{\lambda_{n}^{3 k+1}}\right)
$$

biçiminde yeniden düzenlenebilir. Bu düzenleme $q_{k-1}(x) \in C[0, \pi], k=\overline{1,3}$ fonksiyonları için elde edilecek bir yapılandırma formülü için önemlidir. Dikkat edilirse, burada $\lambda_{n}$ özdeğeri Goktas vd., (2018) çalışmasında hesaplanan özdeğerlerin ifadesinden farklı olarak teorem 2.1'de tanımlanmıştır. Bu nedenle, $l_{j}^{n}$ nin (13) deki ifadesi, Goktas vd., (2018) çalışmasındakine şeklen benzese de tamamen farklıdır. Sonuç olarak, aşağıdaki teorem 3.1 ifadesinde ve ispatında $\lambda_{n}^{3}=n+\frac{1}{2}+O\left(\frac{1}{\sqrt[3]{n}}\right)$ olduğu dikkate alınacaktır.

\section{Potansiyel Fonksiyonlar için Yapılandırma Formülü}

$\mathrm{Bu}$ bölümde, nodal uzunluklar için elde edilen asimptotik formül yardımıyla (1) denkleminin potansiyel fonksiyonlarının belirgin bir formülü verilmiştir.

Teorem 3.1. $q_{k-1}(x) \in C[0, \pi], \quad k=\overline{1,3}$ olsun. Bu durumda, $j=j_{n}(x)=\max \left\{j: x_{j}^{n}<x\right\}$ olmak üzere $n \rightarrow \infty$ iken (1)-(3) probleminin potansiyel fonksiyonları aşağıdaki biçimdedir:

$q_{k-1}(x)=2 \lim _{n \rightarrow \infty}\left(\lambda_{n}^{k+1}-\lambda_{n}^{k-1}+\frac{\pi}{l_{j}^{n}}\left(1-\lambda_{n}\right)\right), \quad k=\overline{1,3}$.

İspat. (13) denklemindeki integraller için ortalama değer teoremi uygulanırsa, 
$l_{j}^{n}=\frac{\pi}{\lambda_{n}^{k}}+\sum_{m=0}^{k-1} \frac{1}{2 \lambda_{n}^{2 k-m}} q_{m}(z) l_{j}^{n}+O\left(\frac{1}{\lambda_{n}^{3 k+1}}\right)$

veya

$$
\sum_{m=0}^{k-1} \lambda_{n}^{m} q_{m}(z)=2\left(\lambda_{n}^{2 k}-\frac{\lambda_{n}^{k} \pi}{l_{j}^{n}}\right)+O\left(\frac{1}{\lambda_{n}^{k+1}}\right)
$$

elde edilir. Burada, $\lambda_{n}$ teorem 2.1 de tanımlanmıştır ve $\left(x_{j}^{n}, x_{j+1}^{n}\right)$ aralığında en az bir $z$ sayısı vardır ki

$\int_{x_{j}^{n}}^{x_{j+1}^{n}} q_{m}(t) d t=q_{m}(z) \cdot l_{j}^{n}$ dir.

$k=1$ için, (14) eşitliğinden

$q_{0}(z)=2\left(\lambda_{n}^{2}-\frac{\lambda_{n} \pi}{l_{j}^{n}}\right)+O\left(\frac{1}{\lambda_{n}^{2}}\right)$

bulunur ki burada $n \rightarrow \infty$ için

$q_{0}(x)=2 \lim _{n \rightarrow \infty}\left(\lambda_{n}^{2}-\frac{\lambda_{n} \pi}{l_{j}^{n}}\right)$

fonksiyonu elde edilir.

Benzer şekilde, $k=2$ için, (14) ve (15) eşitliklerinden

$q_{0}(z)+\lambda_{n} q_{1}(z)=2\left(\lambda_{n}^{4}-\frac{\lambda_{n}^{2} \pi}{l_{j}^{n}}\right)+O\left(\frac{1}{\lambda_{n}^{3}}\right)$

veya

$q_{1}(z)=2\left(\lambda_{n}^{3}-\lambda_{n}+\frac{\pi}{l_{j}^{n}}\left(1-\lambda_{n}\right)\right)+O\left(\frac{1}{\lambda_{n}^{3}}\right)$

bulunur ki burada $n \rightarrow \infty$ için

$q_{1}(x)=2 \lim _{n \rightarrow \infty}\left(\lambda_{n}^{3}-\lambda_{n}+\frac{\pi}{l_{j}^{n}}\left(1-\lambda_{n}\right)\right)$

fonksiyonu elde edilir.

Son olarak, $k=3$ için, (14), (15) ve (16) eşitliklerinden

$q_{0}(z)+\lambda_{n} q_{1}(z)+\lambda_{n}^{2} q_{2}(z)=2\left(\lambda_{n}^{6}-\frac{\lambda_{n}^{3} \pi}{l_{j}^{n}}\right)+O\left(\frac{1}{\lambda_{n}^{4}}\right)$

veya

$q_{2}(z)=2\left(\lambda_{n}^{4}-\lambda_{n}^{2}+\frac{\pi}{l_{j}^{n}}\left(1-\lambda_{n}\right)\right)+O\left(\frac{1}{\lambda_{n}^{4}}\right)$

bulunur ki burada $n \rightarrow \infty$ için

$q_{2}(z)=2 \lim _{n \rightarrow \infty}\left(\lambda_{n}^{4}-\lambda_{n}^{2}+\frac{\pi}{l_{j}^{n}}\left(1-\lambda_{n}\right)\right)$

fonksiyonu elde edilir. Dolayısıyla, teorem 3.1 ispatlanır. 


\section{Sonuç}

Matematiksel fizik problemlerinde kısmi türevli diferensiyel denklemlerle karşılaşılmaktadır. Fiziksel bir sürecin matematiksel bir tanımını yapmak için bu süreci tek olarak belirleyen bazı koşullara da ihtiyaç duyulmaktadır. Bu koşullara, başlangıç veya sınır koşulları denmektedir. Bu tür problemler bazı metotlar yardımıyla öz parametre içeren adi diferensiyel denklemlere dönüştürülmektedir. $\mathrm{Bu}$ denklemlerden biri de SturmLiouville denklemidir. Mevcut çalışmada, SturmLiouville denklemi birden fazla fonksiyon içermesi bakımından klasik Sturm-Liouvile denkleminden farklıdır. Buna ek olarak, problemin sınır koşullarının birinde öz parametre bulunmaktadır. $\mathrm{Bu}$ tür problemler için ters problem çeşitli yöntemlerle araştırılmıştır. $\mathrm{Bu}$ çalışmada ise Prüfer dönüşümü kullanılmıştır. Bu dönüşüm yardımıyla, özdeğerlerin ve nodal parametrelerin asimptotik formülleri elde edilmiştir. $\mathrm{Bu}$ asimptotik formüllerin bilgisiyle, çalışmada ele alınan lineer operatörün biçimi bulunmuştur.

\section{Kaynaklar}

Ambartsumyan, V.A., 1929. Über eine Frage der Eigenwerttheorie. Zeitschrift für Physik, 53, 690-695.

Birkhoff, G. ve Rota, G.C., 1989. Ordinary Differential Equations, 4 edition: Ginn, John Wiley \& Sons, 416p.

Borg, G., 1946. Eine Umkehrung der SturmLiouvilleschen Eigenwertaufgabe. Acta Mathematica, 78(1), 1-96.

Browne, P.J. ve Sleeman, B.D., 1996. Inverse Nodal Problems for Sturm-Liouville Equations with Eigenparameter Dependent Boundary Conditions. Inverse Problems, 12, 377-381.

Chen, Y.T., Cheng, Y. H., Law, C.K. ve Tsay J., 2002. L1 Convergence of the Reconstruction Formula for the Potential Function. Proceedings of the American Mathematical Society, 130, 23192324.

Çakır, A., 2007. Kompleks Potansiyele Sahip SturmLiouville Operatörleri için Ters Saçılma Problemi ve Bazı Uygulamaları. Yüksek Lisans Tezi, Süleyman Demirel Üniversitesi Fen Bilimleri Enstitüsü. Isparta, 58s.

Gel'fand, I.M. ve Levitan, B.M., 1951. On the Determination of a Differential Equation from Its Spectral Function. Izvestiya Akademii Nauk
SSSR Seriya Matematicheskaya, 15(4), 309360.

Goktas S., Koyunbakan H. ve Gulsen T., 2018. Inverse Nodal Problem for Polynomial Pencil of SturmLiouville Operator. Mathematical Methods in the Applied Sciences, 41, 7576-7582.

Guseinov, I.M., Nabiev A.A. ve Pashaev R.T., 2000. Transformation Operators and Asymptotic Formulas for the Eigenvalues of a Polynomial Pencil of Sturm-Liouville Operators. Sibirskii Matematicheskii Zhurnal, 41, 554-566.

Guseinov, I.M. ve Nabiev A.A., 2000. A Class of Inverse Problems for a Quadratic Pencil of Sturm-Liouville Operators. Differentsial'nye Uravneniya, 36(3), 418-420.

Hald, O.L. ve McLaughlin, J.R., 1989. Solutions of Inverse Nodal Problems. Inverse Problems, 5, 307-347.

Hoschtadt, H., 1973. The Inverse Sturm-Liouville Problem. Communications Pure and Applied Mathematics, 26, 715-729.

Kaplan, M., 2019. n-Potansiyel İçeren Sturm-Liouville Operatörü için Ters Nodal Problem. Yüksek Lisans Tezi, Furat Üniversitesi Fen Bilimleri Enstitüsü. Elazığ, 25s.

Keskin, B. ve Özkan, A.S., 2017. Inverse Nodal Problems for Impulsive Sturm-Liouville Equation with Boundary Conditions Depending on the Parameter. Advances in Analysis, 2(3), 151-156.

Koyunbakan, H., 2009. Reconstruction of Potential Function for Diffusion Operator. Numerical Functional Analysis and Optimization, 30(1-2), 1-10.

Koyunbakan, H., 2011. Inverse Problem for a Quadratic Pencil of Sturm-Liouville Operator. Journal of Mathematical Analysis and Applications, 378, 549-554.

Koyunbakan, H. ve Panakhov, E.S., 2007. Half Inverse Problem for Diffusion Operators on the Finite Interval. Journal of Mathematical Analysis and Applications, 326, 1024-1030.

Koyunbakan, H. ve Yilmaz, E., 2008. Reconstruction of the Potential Function and Its Derivatives for the Diffusion Operator. Verlag der Zeitschrift für Naturforch, 63a, 127-130.

McLaughlin, J.R., 1988. Inverse Spectral Theory Using Nodal Points as Data - A Uniqueness Result. Journal of Differential Equations, 73, 354-362.

Nabiev A.A., 2010. On a Fundemental System of Solutions of the matrix Schrödinger Equation with a Polynomial Energy-Dependent Potential. 
Mathematical Methods in the Applied Sciences, 33(11), 1372-1383.

Panakhov, E.S., Koyunbakan, H. ve Ic, U., 2010. Reconstruction Formula for the Potential Function of Sturm-Liouville Problem with Eigenparameter Boundary Condition. Inverse Problems in Science and Engineering, 18 (1), 173-180.

Pinasco, J.P. ve Scarola, C.A., 2015. Nodal Inverse Problem for Second Order Sturm-Liouville Operators with Indefinite Weights. Applied Mathematics and Computation, 256, 819-830.

Shukurov, A. Sh., 2009. The Inverse Problem for a Diffusion Operator. Proceeding of IMM of NAS of Azerbaijan, 30, 105-110.

Şen, E., 2017. A Regularized Trace Formula and Oscillation of Eigenfunctions of a Sturm-
Liouville Operator with Retarded Argument at 2 Points of Discontinuity. Mathematical Methods in the Applied Sciences, 40(18), 7051-7061.

Şen, E., 2018. Computation of Trace and Nodal Points of Eigenfunctions for a Sturm-Liouville Problem with Retarded Argument. Cumhuriyet Science Journal, 39(3), 597-607.

Yang, C. F., 2014. Inverse Nodal Problems for the Sturm-Liouville Operator with a Constant Delay. Journal of Differential Equations, 257(4), 1288-1306.

Yilmaz E. ve Koyunbakan H., 2010. Reconstruction of Potential Function and Its Derivatives for Sturm-Liouville Problem with Eigenvalues in Boundary Condition. Inverse Problems in Science and Engineering, 18(7), 935-944. 